\title{
Is there a link between aging and microbiome diversity in exceptional mammalian longevity?
}

\author{
Graham M Hughes $^{1}{ }^{\text {, John Leech }}{ }^{2}$, Sébastien J Puechmaille ${ }^{3}$, Jose V Lopez ${ }^{4}$, Emma C Teeling ${ }^{\text {Corresp. } 1}$ \\ ${ }^{1}$ School of Biology and Environmental Science, University College Dublin, Dublin, Ireland \\ 2 APC Microbiome Institute, University College Cork, Cork, Ireland \\ 3 Zoology Institute, Ernst-Moritz-Arndt Universität Greifswald, Greifswald, Germany \\ 4 Halmos College of Natural Sciences and Oceanography, Nova Southeastern University, Dania Beach, Florida, United States \\ Corresponding Author: Emma C Teeling \\ Email address: emma.teeling@ucd.ie
}

A changing microbiome has been linked to biological aging in mice and humans, suggesting a possible role of gut flora in pathogenic aging phenotypes. Many bat species have exceptional longevity given their body size and some can live up to ten times longer than expected with little signs of aging. This study explores the anal microbiome of the exceptionally long-lived Myotis myotis bat, investigating bacterial composition in both adult and juvenile bats to determine if the microbiome changes with age in a wild, longlived non-model organism, using non-lethal sampling. The anal microbiome was sequenced using metabarcoding in more than 50 individuals, finding no significant difference between the composition of juvenile and adult bats, suggesting that age-related microbial shifts previously observed in other mammals may not be present in Myotis myotis. Functional gene categories, inferred from metabarcoding data, expressed in the M. myotis microbiome were categorized identifying pathways involved in metabolism, DNA repair and oxidative phosphorylation. We highlight an abundance of 'Proteobacteria' relative to other mammals, with similar patterns compared to other bat microbiomes. Our results suggest that $M$. myotis may have a relatively stable, unchanging microbiome playing a role in their extended 'health spans' with the advancement of age, and suggest a potential link between microbiome and sustained, powered flight. 
1 Is there a link between aging and microbiome diversity in

2 exceptional mammalian longevity?

3 Graham M. Hughes ${ }^{1}$, John Leech², Sébastien J. Puechmaille ${ }^{1,3}$, Jose V. Lopez ${ }^{4}$, Emma C.

4 Teeling ${ }^{1 *}$

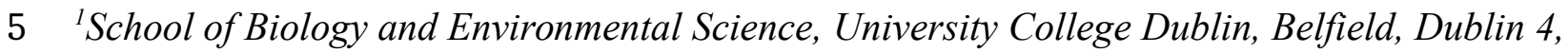

6 Ireland

$7 \quad{ }^{2}$ APC Microbiome institute, University College Cork, Cork, Ireland

$8{ }^{3}$ Zoology Institute, Greifswald University, Soldmann-Str. 14, D-17489 Greifswald, Germany

$9{ }^{4}$ Halmos College of Natural Sciences and Oceanography, Nova Southeastern University, Dania 10 Beach FL 3304 Florida, USA

11 Graham M. Hughes: g.hughes@ucd.ie

12 John Leech: john.leech@teagasc.ie

13 Sébastien J. Puechmaille: s.puechmaille@gmail.com

14 Jose V. Lopez: joslo@nova.edu

$15 *$ Emma C. Teeling, corresponding author: emma.teeling@ucd.ie 
16

17

18

19

20

21

22

23

24

25

26

27

28

29

30

31

32

\section{Abstract}

A changing microbiome has been linked to biological aging in mice and humans, suggesting a possible role of gut flora in pathogenic aging phenotypes. Many bat species have exceptional longevity given their body size and some can live up to ten times longer than expected with little signs of aging. This study explores the anal microbiome of the exceptionally long-lived Myotis myotis bat, investigating bacterial composition in both adult and juvenile bats to determine if the microbiome changes with age in a wild, long-lived non-model organism, using non-lethal sampling. The anal microbiome was sequenced using metabarcoding in more than 50 individuals, finding no significant difference between the composition of juvenile and adult bats, suggesting that age-related microbial shifts previously observed in other mammals may not be present in Myotis myotis. Functional gene categories, inferred from metabarcoding data, expressed in the $M$. myotis microbiome were categorized identifying pathways involved in metabolism, DNA repair and oxidative phosphorylation. We highlight an abundance of 'Proteobacteria' relative to other mammals, with similar patterns compared to other bat microbiomes. Our results suggest that $M$. myotis may have a relatively stable, unchanging microbiome playing a role in their extended 'health spans' with the advancement of age, and suggest a potential link between microbiome and sustained, powered flight.

\section{Introduction}

The importance of the gut microbiome, the collection of microflora or microbiota inhabiting various regions of the gastro-intestinal tract, has become apparent in recent years. It is known to facilitate the fermentation of nutrients, such as carbohydrates, into short chain fatty acids (e.g. butyrate) for use by the host (Flint et al., 2012). Many vitamins are also synthesized by the microbiome such as vitamin B7, B12 and Vitamin A, which may be otherwise unavailable (O'Hara and Shanahan, 2006). In addition to nutrition, more evidence is accumulating pointing to "microbiome-wide associations" with health and disease in humans and other hosts (Gilbert et al., 2016), and the composition of the mammalian gut microbiome has been linked to the process of biological aging (Biagi et al., 2016; Mello et al., 2016). Aging is characterized by the progressive decline of function, increased frailty and an increase in chronic disease (López-Otín et al., 2013). Studies of the human microbiome have reported shifts in microbial composition across different stages of life, with a high degree of variability at the two extremes of infancy and old age (Saraswati and Sitaraman, 2015). A shift from a microbiome that deals exclusively with breast milk in nursing infants to a more diverse microbiome that can metabolize a wider range of nutrition (Yatsunenko et al., 2012) is observed in early to middle stages of life. In later life, a reduction of lactobacilli and an increase of potentially pathogenic Enterobacteriaceae have been observed in frail individuals (van Tongeren et al., 2005). This accumulation of pathogenic flora has been associated with a range of clinical problems such as infection, cancer and deficiencies in immune response (Atarashi et al., 2013; Saraswati and Sitaraman, 2015). Similar microbial shifts are observed in mice, such as the decrease in bacteria that synthesize vitamin B12 in older age cohorts, leading to overall changes in microbiome composition and function in age related frailty (Langille et al., 2014) implying a general trend in the aging gut. The question of whether or not such microbial shifts are a symptom rather than a driver of aging has yet to be conclusively answered.

Bats are exceptional mammals not only because of their capability of powered flight but also due to the diverse range of life histories they exhibit, with exceptional longevity being of 
60

61

62

63

64

65

66

67

68

69

70

71

72

73

74

75

76

77

78

79

80

81

82

83

84

85

86

87

88

89

90

91

92

93

94

95

96

97

98

99

100

101

102

103

104

particular interest (Austad, 2010). Within bats, a number of species in the family Vespertilionidae demonstrate extreme longevity, living up to ten times longer than expected given their body size (Austad, 2010; Shen et al., 2010). Surviving in the wild requires maintaining agility; speed and high frequency hearing to capture prey on a daily basis. Therefore, a long lifespan in bats coincides with a long health-span. Elucidating the changes that occur in microbial composition over time in these exceptionally long-lived organisms will shed light on the role of the microbiome in extended health-spans. While previous studies of bat microbiomes have focused on the effects of phylogeny and diet, this is the first bat microbiome study to focus on aging.

In this study, we have used DNA metabarcoding of the 16S rRNA gene and highthroughput sequencing to characterize the structure and function of the anal microbiome from 52 wild, exceptionally long-lived insectivorous Myotis myotis bats (maximum lifespan (MLS) 37 years (Gaisler et al., 2003)) using a non-lethal sampling method. Myotis myotis makes an ideal comparison to current mouse microbiome models as these bats are similar in body size but can live up to 32 years longer. We show that there is no obvious shift in bacterial composition between juvenile and the early stages of adulthood in Myotis myotis, as has been observed in other mammals. We find that the highest number of pathways expressed in bat metagenomes are involved in metabolism, energy consumption, DNA repair and oxidative phosphorylation, all reactions that may play a role in the use of powered flight and aging, suggesting a potential interaction between long-term microbiome stability, lifespan and powered flight. A microbiome that does not change over time may have profound effects on age-related infections and immune deficiencies, opening up new avenues of microbiome research in non-model organisms. Finally, we compare the relative abundance of major bacterial phyla in M. myotis with those of other bats and mammals, highlighting the high abundance of 'Proteobacteria' in Chiroptera relative to other mammals.

\section{Materials and Methods}

\section{Sample collection}

All field procedures were carried out in accordance with the ethical guidelines and permits delivered by 'Arrêté' by the Préfet du Morbihan, Bretagne awarded to Eric Petit, Frédéric Touzalin and Sébastien Puechmaille for the time period 15 June-15 September 2013-2017. Full ethics approval and permission (AREC-13-38-Teeling) for capture and field sampling was awarded by the University College Dublin ethics committee. Myotis myotis were sampled in western France, (Brittany), July 2013, from four large roost locations: Béganne (Beg), Férel (Fer), La Roche-Bernard (LRB) and Noyal-Muzillac (NM). The maximum distance between these four sites is roughly $105 \mathrm{~km}^{2}$. Bats were caught in custom harp traps while leaving their roost (hence typically before foraging/feeding) and were initially placed in individual cloth bags (Huang et al 2016). Each bat was identified by a unique transponder inserted under the skin when the bat was first captured. If captured and transponded as a juvenile ( $\sim<6$ weeks old), indicated by the lack of fused finger bones, the exact age at recapture was known. Validated weaning status could not be determined for juvenile bats, however the sampled juveniles were capable of flight, suggesting that the majority were partially weaned, but opportunistic suckling cannot be ruled out. If caught and transponded for the first time as an adult, years since first capture was noted and a plus sign indicated the individual was older than age estimated from first capture. For each bat, a Copan FLOQSwab ${ }^{\mathrm{TM}}$ swab was gently inserted into the anus and removed. The swab was then placed into an Eppendorf and immediately flash frozen in liquid nitrogen. Swabs were subsequently place in ethanol during transport for sequencing. 
106

107

108

109

110

111

112

113

114

115

116

117

118

Sample age cohorts ranged from ' 0 years' to ' $4+$ years', with individuals whose exact age was unknown but minimum age could be determined denoted by ' + '. To investigate differences in anal flora between juvenile and adult bats, samples were categorized into a number of age data sets. Age dataset 1 contained known ages only $(\mathrm{n}=29)$. When comparing adults and definitive juveniles, age dataset 2 categorized bats that were 1 or more years old as adults $(n=33)$, while 0 year olds were considered juvenile $(n=19)$. In age dataset 3 , the cut-off for juvenile was increased from 0 to 1 year (juvenile $\mathrm{n}=27$ ), to investigate if the microbiome of a 1 year old M. myotis bat transitioning from juvenile to adult had an influence on any age associated microbial shifts that might be observed. For age dataset 3, ' $1+$ ' individuals were excluded. Finally, age dataset 4 contained all ages as individual categories (Table 1). Juvenile to adult age ranges cover the entire lifespan of a mouse or similar sized mammal (0-4 years). In addition to age, bat samples were categorized based on gender and location of collection (sample site was not catalogued for two individuals, MMY247 and MMY51, which were instead referred to as 'site undefined').

\section{$16 S$ rRNA library preparation and sequencing}

Total microbial genomic DNA from each swab sample was extracted using MO BIO's PowerLyzer ${ }^{\mathrm{TM}}$ PowerSoil ${ }^{\mathbb{}}$ kit, following Earth Microbiome (earthmicrobiome.org) standards and recommendations. Following extractions, DNA quality was checked via agarose gel electrophoresis. Purity of the $16 \mathrm{~S}$ rRNA amplicons after adding barcodes was determined via a 2200 TapeStation Bioanalyzer (Agilent Technologies), while quantity of each DNA (4 pM of each sample) was measured with a Qubit 2.0 flourometer (Life Technologies) prior to each MiSeq run. The protocol detailed in Caporaso et al. (2012), using the V4 hyper-variable region primers $515 \mathrm{~F} / 806 \mathrm{R}$ for paired-end $16 \mathrm{~S}$ rRNA sequencing was strictly followed.

Libraries were generated for all 52 samples using the Illumina Nextera XT dual primer protocol and 16S metagenomic library prep guide. Blank extractions containing no DNA, and tested with barcoded and non-barcoded index primers, were used as a negative control to investigate potential contamination during laboratory procedures. Samples were sequenced using the MiSeq platform and 250bp paired end chemistry. Taxonomic analysis was carried out using the Quantitative Insights into Microbial Ecology (QIIME) suite of software, version 1.9.1 (Caporaso et al., 2010). Paired end reads were filtered using a phred score threshold of 30, and chimeric sequences were removed using the QIIME 'split_libraries_fastq.py' and 'filter_fasta.py' scripts. Reads from each sample were then overlapped, joining the forward and reverse reads, using the fastq-join method as implemented in QIIME.

\section{OTU Picking and diversity analyses}

Overlapped sequences from each bat sample were used for Operational Taxonomic Unit (OTU) picking. OTU picking involves the process of aligning sequences found in each sample to a reference database and assigning them to an OTU (a cluster of similar reads representing a bacterial phyla, genera or species, depending on level of resolution). For this study, the Greengenes annotated 16S reference database (DeSantis et al., 2006) and PyNast aligner was used, with a minimum sequence clustering identity of $97 \%$ (Caporaso et al., 2010). Greengenes was chosen as our reference database as it has been previously used to characterize other bat microbiomes, which are included in our study (Philips et al., 2012; Carillo-Araujo et al., 2015). The method of 'open OTU picking', where reads that do not map to a reference database are subsequently clustered de novo, was applied. 
149

150

151

152

153

154

155

156

157

158

159

160

161

162

163

164

165

166

167

168

169

170

171

172

173

174

175

176

177

178

179

180

181

182

183

184

185

186

187

188

189

190

191

192

193
The mean OTU abundance within each sample was calculated as alpha diversity in QIIME. Samples were rarefied to 8000 reads, removing possible biases introduced due to uneven sequencing depth, using the 'core_diversity_analysis.py' script, with parameter '-e' set to 8000. This minimum was chosen to include as many samples as possible while also providing enough depth to get a good representation of the microbiome. Despite potentially reduced statistical power (McMurdie and Holmes, 2014), rarefaction was chosen as the ideal means of data normalization given the range of reads across samples (Weiss et al., 2017), resulting in abundances comparable to other mammalian microbiomes (see below). A change in alpha diversity has previously been used to differentiate between young, mature and old mammals (Yatsunenko et al., 2012; Frese et al., 2015), hence alpha-diversity was calculated across each pre-defined age dataset, in addition to gender and location of collection. The mean phylogenetic diversity (PD), a measure of alpha diversity accounting for phylogenetic differences between bacterial species (Faith, 1992), was calculated for each sample and metadata category. This was compared to the gut flora of previously characterized members of Vespertilionidae (Philips et al., 2012), to determine if the anal flora is representative of the gut microbiome, further implying the utility of our sampling method. Beta diversity (abundance between samples) was also calculated across all samples before and after rarefaction using both weighted and un-weighted Unifrac distance matrices, which are based on differences between samples using phylogenetic information (Lozupone and Knight, 2005). Hierarchical clustering, using Ward's method (Ward, 1963), based on number of OTUs detected was applied to samples using $R$ to visualize any separation based on age, sex or location that could be observed. The core microbiome was computed as bacterial species present in at least a 'user-specified' percentage of samples. Minimum thresholds ranging from 50-80\% were investigated across each age dataset, gender and location.

\section{Statistical analysis}

PCoA plots were generated based on the weighted Unifrac distance matrices of beta-diversity for each different categorical variable. These plots were visualized to determine if linear separation existed between ages, gender and location of sample collection. Additionally, Analysis of Similarity (ANOSIM) was applied to the weighted Unifrac distance matrices to determine if a statistically significant difference existed for each data category. ANOSIM is non-parametric and was made distribution-free by using a number of permutations (9999).

OTU frequencies between juvenile and adult samples were compared using a KruskalWallis analysis of variance to identify bacteria whose abundance was significantly different across categories, using the 'group_significance.py' script in QIIME, with Bonferroni correction applied across all results. Additionally, Similarity Percentage (SIMPER; Clarke, 1993) analysis, a method to assess taxa responsible for the overall observed similarity/dissimilarity between groups, was carried out on the pooled OTU abundance counts for each location both before and after rarefaction. SIMPER was carried out using the PAST software (Hammer et al., 2001).

\section{Predictive characterization of microbiomes (PICRUSt)}

Phylogenetic Investigation of Communities by Reconstruction of Unobserved States (PICRUSt; Langille et al., 2013) was used to predict genes/ pathways that might be expressed in bacteria found in the anus of $M$. myotis. Based on bacterial species whose protein coding genes are described in the KEGG and COG databases, PICRUSt attempts to infer genes expressed in each OTU using phylogenetic similarity to a previously characterized species. As per its operational requirements, PICRUSt was used on a normalized biom table generated using the 'closed OTU 
194 picking' method in QIIME, excluding reads that could not be mapped to a reference OTU cluster.

195 To investigate if metagenomic changes occurred between juvenile and adult microbiomes,

196 functional analysis was conducted using the methodologies described by Phillips et al. (2017),

197 implemented in the R package 'FunkyTax'. This analysis was used to identify if predicted

198 metagenomic function remained the same, were enhanced (increase in abundances of

199 contribution across age groups) or divergent (significantly different composition across ages for a

200 predicted function)

201 Phylogenetic comparison to other mammals

202 The average relative abundances of 9 bacteria phyla in M. myotis were compared to available 203 microbiome data from other mammals, focusing specifically on the gut, rectal or faecal samples, 204 to investigate if closely related taxa shared similar composition. Bacterial abundances were 205 compared under the assumption that, while based on different experimental conditions and 206 research goals, each mammalian microbiome study represents an accurate abundance calculation 207 for that species given the experimental setup. The relative abundances of 35 additional bat 208 species (Philips et al., 2012; Carrillo-Araujo et al., 2015), cow rectum (Mao et al., 2015), dolphin 209 rectum (Bik et al., 2016), dog faecal sample (Swanson et al., 2011), human and mouse faecal 210 sample (Krych et al., 2013), gorilla faecal sample (Gomez et al., 2015) and Tasmanian devil 211 faecal samples (Cheng et al., 2015) were compared using principal component analysis (PCA). $212 K$-means clustering, with the optimum $k$ number of clusters decided using the elbow criterion, 213 was carried out in $R$ and used to identify if any 'bat-specific' clusters were present, and if species 214 clustering resembled phylogeny (Datzmann et al., 2010; Meredith et al., 2011; Philips et al., 215 2012).

216 Results

217 QIIME analyses

218 Across all 52 samples, 2,947,977 read pairs were overlapped and used for downstream analyses

219 (Table 1). No spurious amplifications were detected in the negative controls. A rarefying 220 threshold of 8000 sequences reduced the final number of $M$. myotis samples to 41 individuals.

221 The number of unique OTUs per sample ranged from 77 to 747 (mean=299). No age, sex or

222 location-specific clusters were observed across samples using hierarchical clustering

223 (Supplementary Figure 1). Mean alpha diversity (diversity per sample; PD) for M. myotis was

224 22.34. Similar PD values were found between male (20.4) and female (23.85) and between

225 juveniles and adults (Figure 1 (a), (b); Table 2), with neither comparison showing significant

226 differences (Kruskal-Wallis test, $\mathrm{p}>0.05$ ). Mean PD values for each data category are displayed in

227 Table 2, with additional bacterial abundances displayed in Supplementary Figure 2 and

228 Supplementary Table 1.

229

230

231

232

233

234

235

A total of 47 OTUs were present in $50 \%$ of all samples consisting of the Actinobacteria, Chlamydiae, Cyanobacteria, Firmicutes, Proteobacteria and Tenericutes phyla highlighting some inter-individual OTU diversity (Supplementary Figure 3), with Proteobacteria and Chlamydiae/Tenericutes having the highest and lowest abundance of OTUs, respectively. When increasing this core microbiome threshold, 13 OTUs, containing Ureibacillus (Firmicutes), Corynebacterium (Actinobacteria), Enterococcus (Firmicutes) and Pseudomonas (Proteobacteria; Table 3, Supplementary Table 2, Supplementary Figure 4), were found present in $80 \%$ of all bats.

236

Statistical Analyses 
237 Principal coordinate analysis showed no clear observable separation of samples based on age,

238 gender or collection site for both un-weighted and weighted Unifrac distances. Weighted Unifrac

239 distances explained a greater percentage of variance using three principal coordinates both before

240 rarefaction $(55.27 \%$ variance, Supplementary Figures 5, 6) and after rarefaction $(60.59 \%$

241 variance, Figure 2 (a), (b); Supplementary Figure 7). Using ANOSIM, no significant differences

242 were found after Bonferroni correction, reflected in the low R test-statistics $(\mathrm{R} \leq 0.14$; Chapman

243 and Underwood, 1999) in all cases. A Kruskal-Wallis non-parametric ANOVA found no

244 significant differences between male and female bats, ruling out gender as a mode of microbial

245 diversity. OTUs were also compared between adult and juveniles (age dataset 2, 3) using a

246 Kruskal-Wallis test revealing 4 OTUs from the common GI-inhabiting family Helicobacteraceae

247 showing differential abundances after Bonferroni correction $(\mathrm{p}<0.05)$ in age dataset 2 , and no

248 differentially represented OTUs observed between juvenile and adult bats for age dataset 3.

249

250

251

252

253

254

255

256

257

258

259

260

261

262

263

264

265

266

267

268

269

When comparing the percentage dissimilarity of OTU abundance between sites, the average dissimilarity across sites for phyla present was $52.68 \%$ and $53.79 \%$ before and after rarefaction, respectively. At this taxonomic level, the phyla that contributed most to the overall dissimilarity were Firmicutes (mean dissimilarity $15.09 \%$ before and $16.68 \%$ after rarefaction) and Proteobacteria (mean dissimilarity 14.83 before and $16.45 \%$ after rarefaction). Dissimilarity increased for each taxonomic level, with $80.42 \%$ and $85.3 \%$ dissimilarity at the family level (Supplementary Table 3). This dissimilarity may reflect the absence of specific OTUs in one site relative to another, however such dissimilarity did not have a strong affect overall when comparing Unifrac distances, as site-specific clusters could not be determined using PCoA (Supplementary Figure 6,7). Only one OTU, from the phylum Actinobacteria, showed differential abundances between locations (ID: 179312; $p=0.0002$ ) suggesting collection site did not have a significant effect on microbial composition.

\section{PICRUSt and phylogenetic comparison to other mammals}

Across all samples, PICRUSt inferred a total of 41 KEGG pathways present in the M. myotis anal flora (Supplementary Table 4; Figure 3). Of these 41 pathways, the majority of genes and their top 2 pathways belonged to membrane transport (14.24\%; 'transporters' and 'ABC transporters'), carbohydrate transport (10.51\%; 'amino sugar and nucleotide sugar metabolism' and 'glycolysis / gluconeogenesis'), amino acid metabolism (9.35\%; 'amino acid related enzymes' and 'arginine and proline metabolism'), replication and repair (7.62\%; 'DNA repair and recombination proteins' and 'chromosome') and energy metabolism (5.21\%; 'oxidative phosphorylation' and 'carbon fixation pathways in prokaryotes').

270

271

272

273

274

275

276

277

278

279

When comparing predicted metagenomic function between juvenile and adult bats, it was found that out of 2330 KEGG pathways analyzed, 1713 predicted functional categories were 'enhanced' between juvenile and adult bats for age dataset 2, with a large number of 'metabolic processes' in both the enhanced (726) and divergent (246) classification across age groups (Supplementary Table 5 (a)). Enhanced categories indicate that the functional frequency, rather than the contributing microbiome community, differs across age while divergence implies both the function and contributing community are different. Only enhanced functions were found for age dataset 3, the majority of which were involved in metabolism (Supplementary table 5 (b)).

A comparison of 44 mammal microbiomes, including M. myotis, highlighted high abundances of Proteobacteria, but lower abundances of Bacteroidetes, in bats compared to 280 terrestrial mammals (Figure 4). These data were analyzed and visualized using a PCA plot to 281 investigate if closely related species shared similar microbial composition (76.8\% variance; 
282 Supplementary Figure 8). Using a $k$ value of 4, two clusters composed almost exclusively of bats 283 (Supplementary Table 6) were identified. Separation between bats and other mammals was 284 apparent, with a large number of bat samples driven by the presence of Proteobacteria (Cluster 3; 285 Supplementary Table 6), despite differing diets (Figure 4).

\section{Discussion}

287 When analyzing the anal microbiome of Myotis myotis, phylogenetic diversity was slightly higher 288 (22.34) than the PD of the gut microbiome previously established in Vespertilionidae (19.055;

289 Philips et al., 2012). As these data were obtained through non-lethal sampling, this approach will 290 allow re-sampling of the same individual each year. It is therefore an extremely useful tool for 291 longitudinal microbiome studies. The mean number of observed OTUs was similar between 0 292 and 1 year olds (age dataset 1) and did not reflect increases observed for similar ages in humans 293 (Yatsunenko et al., 2012). An increase in gut flora diversity between nursing and weaning 294 juveniles has also been observed in pigs (Frese et al., 2015). It was not possible to determine 295 weaning/nursing status of juvenile bats sampled in this study, however, as all juveniles were 296 flying, there were likely partially weaned.

In their study of the aging mouse microbiome, Langille et al. (2014) observed a clear and statistically significant separation between young ( 0.48 years), middle (1.61 years) and old-aged (2.35 years) mice based on gut flora. Using human data, Yatsunenko et al. (2012) and Biagi et al. (2016) identified clustering patterns based on a variety of age ranges, spanning 0-83 and 22-109 years old, implying a general trend in aging. Using similar methods, despite some variability across individuals, we were unable to identify any clear separation between juvenile and adults in $41 \mathrm{M}$. myotis bats, suggesting an element of stability in microbial diversity. The oldest definitive age in our sample cohort is 4 years, representing the MLS of a mouse, a similar sized mammal. The lack of samples representing the late stages of the 37-year lifespan precludes inferences about microbiome composition in the oldest $M$. myotis bats relative to juvenile and adult bats. However, if the microbiome of $M$. myotis remains stable or static throughout their life, it is expected that the abundances of flora in 'old' individuals will be similar to the juveniles and adults described here, further implying microbiome stability as they age. This would emphasize the importance of microbiome stability in longer health-spans, resembling the fine tuned level of microbiome homeostasis regulating host aging in C. elegans (Han et al., 2017). Our intention in future studies is to involve older M. myotis bats to further elucidate the potential role of the microbiome in exceptional longevity and to what extent microbial variability between samples affects global microbiome patterns in bats.

The finding of dominant and variable abundances of Firmicutes and Actinobacteria is consistent with previous mammalian microbiome profiles (Ley et al., 2008). The genera identified in $80 \%$ of samples belong to families with many pathogenic species. As these taxa could not be identified to species or strain level, no conclusions can be drawn regarding their presence in the core M. myotis microbiome. Despite differences in OTUs found across sites, no significant effect of locality and thus roost specificity could be established. Bacteria from the genera Citrobacter, Enterobacter, Escherichia, Klebsiella, Proteus and Streptococcus were found present in a number of samples, and have previously been documented in the feces of M. myotis in Italy (Di Bella et al., 2003). A study of the anal microflora in M. myotis in Poland identified species from the Lactobacillus, Enterococcus, Serratia, Corynebacterium and Pseudomonas genera (Rózalska et al., 1998), all of which are represented in samples included here, indicating a common core of flora, independent of location. A common microbial core is similar to that 
327 observed in ruminants (Henderson et al., 2016), and contrasts to the social effects of microbial

328 diversity observed in wild baboons (Tung et al., 2015).

329

330

331

332

333

334

335

336

337

338

339

340

341

342

343

344

345

346

347

348

349

350

351

352

353

354

355

356

357

358

359

360

361

362

363

364

365

366

367

368

369

370

371

372

The characterization and comparison of metagenomic content in the anal microbiome determined that the frequency of a number of known functional categories, of which a large proportion were involved in general metabolism, were enhanced from juveniles to adults. There was some divergence observed for age dataset 2, implying some difference in functional abundances across certain samples (1 year old). Other categories involved in energy consumption, DNA repair and oxidative phosphorylation were also present and enhanced and may imply a role of bat gut flora in enabling them to counteract the deleterious effects of metabolically costly powered flight (Shen et al., 2010). Studies of metabolism in insectivorous bats have determined that flight is fuelled directly by ingested prey (insects from the families Carabidae, Orthoptera, Diptera and Arachnida in M. myotis (Di Bella et al., 2003)) immediately after consumption, implying extremely rapid metabolism (Voight et al., 2008; Voight et al., 2010). This has also been observed in nectivorous and frugivorous bats (Voight and Speakman, 2007; Amitai et al., 2010). A high abundance of bacteria producing enzymes involved in energyrelated pathways might contribute to the overall managing of, and coping with, by-products of such high metabolism in M. myotis. If this is the case, a relatively stable microbial community, as observed between adults and juveniles, may not only be involved in extended longevity, but also play an important role in sustained flight.

By comparing microbiome content across 44 mammals, certain 'bat-specific' clustering was observed. However, without longitudinal data, investigating if the abundance of bacteria in other bats species changes with age, like humans and mice, is not possible at this time. An abundance of 'Proteobacteria' was observed in multiple different bat species, including $M$. myotis. Interestingly, Proteobacteria also show high abundances in birds (Hird et al., 2015), suggesting this phylum or the metabolic byproducts it produces, may have a putative role in flight and its metabolic costs rather than aging. As the abundances of the bacteria phyla in the $M$. myotis microbiome appear similar to other bat compositions, it is possible that similar gene pathways are expressed across Chiropteran metagenomes, implicating a putative role of the microbiome in dealing with the energetic demands of flight. Terrestrial, non-chiropteran species had a much larger abundance of Bacteroidetes relative to bats. The reported low abundance of the Bacteroidetes phylum in the bat gut is consistent with the same results from bat skin (Avena et al., 2016).

\section{Conclusion}

Previous studies investigating the role of the microbiome in aging have focused on human and mice samples, and have demonstrated correlations between microbial changes and a pathogenic aging phenotype. Given the exceptional long life and health-span observed in certain species of bats given their body size, we have investigated the microbiome of the long-lived M. myotis using juvenile and early adulthood samples, covering the lifespan range of a similar sized mouse, acquired using a non-lethal mode of sampling. Consistent with earlier studies of bat gut flora, we find a high level of Proteobacteria and Firmicutes, with the microbiome possibly contributing to metabolism, DNA replication/ repair and oxidative phosphorylation. Despite some variability across samples, distinct differential abundances in bacterial composition between adult and juvenile bats were not found, contrasting to patterns observed in humans and mice. Instead we observe an element of microbiome stability between juvenile and adult $M$. myotis bats. Given the KEGG pathways present in the anal bat flora, it is possible that metabolites produced by the bat microbiome enable them better tolerate the damaging bi-products of flight and may increase 
373 metabolic efficiency, with downstream affects on aging. Future studies of older M. myotis age 374 cohorts will determine whether patterns observed here continue into old age, and to what extend 375 inter-individual variability affects global microbiome patterns in bat species. Such comparative 376 microbiome studies will help further elucidate bacterial phyla that may contribute to healthier 377 aging.

\section{Acknowledgements}

379 We thank Nicole Foley, David Jebb, Serena Dool, Eric Petit, Frédéric Touzalin, Olivier Farcy and 380 Arnaud Le Houédec, and the numerous volunteers and students from BV and University College 381 Dublin for their extensive help in the field, sample collection and the owners/local authorities for 382 allowing access to the sites. We thank Renee Potens, Nidhi Vijayan and Jorie Skutas at NSU for 383 assistance with molecular sample processing and Cole Easson for his help with read processing.

\section{References}

385 Amitai O, Holtze S, Barkan S, Amichai E, Korine C, Pinshow B, et al. (2010). Fruit bats 386 (Pteropodidae) fuel their metabolism rapidly and directly with exogenous sugars. Journal of 387 Experimental Biology 213: 2693-2699.

388 Atarashi K, Tanoue T, Oshima K, Suda W, Nagano Y, Nishikawa H, et al. (2013). Treg induction 389 by a rationally selected mixture of Clostridia strains from the human microbiota. Nature 500:

$390 \quad 232-236$.

391 Austad SN. (2010). Methusaleh's Zoo: How nature provides us with Clues for Extending Human 392 Health Span. Journal of Comparative Pathology 142: S10-S21.

393 Avena CV, Parfrey LW, Leff JW, Archer HM, Frick WF, Langwig KE, et al. (2016).

394 Deconstructing the Bat Skin Microbiome: Influences of the host and the Environment. Frontiers 395 in Microbiology 7: 1753. 
396 Biagi E, Franceschi C, Rampelli S, Severgnini M, Ostan R, Turroni S, et al. (2016). Gut

397 microbiota and Extreme Longevity. Current Biology 26: 1480-1485.

398 Bik EM, Costello EK, Switzer AD, Callahan BJ, Holmes SP, Wells RS, et al. (2016). Marine

399 mammals harbor unique microbiotas shaped by and yet distinct form the sea. Nature

400 Communications 7: 10516.

401 Caporaso JG, Kuczynski J, Stombaugh J, Bittinger K, Bushman FD, Costello EK, et al. (2010).

402 QIIME allows analysis of high-throughput community sequence data. Nature Methods 7: 335-

403336.

404 Caporaso JG, Lauber CL, Walters WA, Berg-Lyons D, Huntley J, Fierer N, et al. (2012). Ultra-

405 high-throughout microbial community analysis on the Illumina HiSeq and MiSeq platforms.

406 ISME Journal 6: 1621-1624.

407 Carrillo-Araujo M, Tas N, Alcántara-Hernández RJ, Gaona O, Schondube JE, Medellén RA, et al. 408 (2015). Phyllostomid bat microbiome composition is associated to host phylogeny and feeding 409 strategies. Frontiers in Microbiology 6: 1-9.

410 Chapman MG, Underwood AJ. (1999). Ecological patterns in multivariate assemblages:

411 information and interpretation of negative values in ANOSIM tests. Marine Ecology Progress

412 Series 180: 257-265.

413 Cheng Y, Fox S, Pemberton D, Hogg C, Papenfuss AT, Belov K. (2015). The Tasmanian devil 414 microbiome-implications for conservation and management. Microbiome 3: 76.

415 Clarke KR. (1993). Non-parametric multivariate analysis of changes in community structure. 416 Australian Journal of Ecology 18: 117-143.

417 Datzmann T, Helversen O, Mayer F. (2010). Evolution of nectarivory in phyllostomid bats 418 (Phyllostomidae Gray, 1825, Chrioptera: Mammalia). BMC Evolutionary Biology 10: 165.

419 DeSantis TZ, Hugenholtz P, Larsen N, Rojas M, Brodie EL, Keller K, et al. (2006). Greengenes, 420 a chimera-checked 16S rRNA gene database and workbench compatible with ARB. Applied 421 Environmental Microbiology 72: 5069-5072.

422 Di Bella C, Piraino C, Caracappa S, Fornasari L, Violani C, Zava B. (2003). Enteric Microflora in 423 Italian Chiroptera. Journal of Mountain Ecology 7: 221-224.

424 Faith DP. (1992). Conservation evaluation and phylogenetic diversity. Biological Conservation 425 61: 1-10.

426 Flint HJ, Scott KP, Louis P, Duncan SH. (2012). The role of the gut microbiota in nutrition and 427 health. Nature Reviews Gastroenterology \& Hepatology 9: 577-589.

428 Frese SA, Parker K, Calvert CC, Mills DA. (2015). Diet shapes the gut microbiome of pigs 429 during nursing and weaning. Microbiome 3: 28.

430 Gaisler J, Hanak V, Hanzal V, Jarsky V. (2003). Results of bat branding in the Czech and Slovak 431 Republics, 1948-2000. Vespertilio 7: 3-61. 
432 Gilbert JA, Quinn RA, Debelius J, Xu ZZ, Morton J, Garg N, et al. (2016). Microbiome-wide 433 association studies link dynamic microbial consortia to disease. Nature 535: 94 - 103.

434 Gomez A, Petrzelkova K, Yeoman CJ, Vlckova K, Mrázek J, Koppova I, et al. (2015). Gut 435 microbiome composition and metabolomic profiles of wild western lowland gorillas (Gorilla 436 gorilla gorilla) reflect host ecology. Molecular Ecology 24: 2551-2565.

437 Han B, Sivaramakrishnan P, Lin C-CJ, Neve IAA, He J, Wei L, et al. (2017). Microbial Genetic 438 Composition Tunes Host Longevity. Cell 7: 1249-1262.

439 Hammer Ø, Harper DAT, Ryan PD. (2001). PAST: Paleontological statistics software package for 440 education and data analysis. Paleontologia Electronica 4: 1-9.

441 Henderson G, Cox F, Ganesh S, Jonker A, Young W, Global Rumen Census Collaborators, 442 Janssen PH (2016). Rumen microbial community composition varies with diet and host, but a 443 core microbiome is found across a wide geographical range. Scientific Reports 5: 14567.

444 Hird SM, Sánchez C, Carstens BC, Brumfield RT. (2015). Comparative Gut microbiota of 59 445 Neotropical Bird Species. Frontiers in Microbiology 6: 1403.

446 Huang Z, Gallot A, Lao NT, Puechmaille SJ, Foley NM, Jebb D, et al. (2016). A nonlethal 447 sampling method to obtain, generate and assemble whole blood transcriptomes from small, wild 448 mammals. Molecular Ecology Resources 16: 150-162.

449 Krych L, Hansen CHF, Hansen AK, van den Berg FWJ, Nielsen DS. (2013). Quantitatively 450 Different, yet Qualitatively Alike: A Meta-Analysis of the Mouse Core Gut Microbiome with a 451 View towards the Human Gut Microbiome. PLoS ONE 8: e62578.

452 Langille M, Zaneveld J, Caporaso JG, McDonald D, Knights D, Reyes J, et al. (2013). Predictive 453 functional profiling of microbial communities using 16S rRNA marker gene sequences. Nature 454 Biotechnology 31: 814-821.

455 Langille M, Meehan CJ, Koenig JE, Dhanani AS, Rose RA, Howlett SE, et al. (2014). Microbial 456 shifts in the aging mouse gut. Microbiome 2: 50.

457 Ley RE, Lozupone CA, Hamaday M, Knight R, Gordon JI (2008). Worlds within worlds: 458 evolution of the vertebrate gut microbiota. Nature Reviews Microbiology 6 (10): 776-788.

459 López-Otín C, Blasco MA, Partridge L, Serrano M, Kroemer G. (2013). The hallmarks of aging. 460 Cell 153: 1194-1217.

461 Lozupone C, Knight R. (2005). UniFrac: a New Phylogenetic Method for Comparing Microbial 462 Communities. Applied Environmental Microbiology 71: 8228-8235.

463 Mao S, Zhang M, Liu J, Zhu W. (2015). Characterising the bacterial microbiota across the 464 gastrointestinal tracts of dairy cattle: membership and potential function. Scientific Reports $\mathbf{5}$ : 46516116.

466 McMurdie PJ, Holmes S. (2014). Waste Not, want Not: Why rarefying Microbiome Data is 467 Inadmissable. PLoS Computational Biology 10: e1003531. 


\section{Manuscript to be reviewed}

468 Mello AM, Paroni G, Daragjati J, Pilotto A. (2016). Gastrointestinal microbiota and their

469 contribution to healthy aging. Digestive Diseases 34: 194-201.

470 Meredith RW, Janečka JE, Gatesy J, Ryder OA, Fisher CA, Teeling EC, et al. (2011). Impacts of 471 the Cretaceous Terrestrial Revolution and KPg extinction on mammal diversification. Science 472 334: 521-524.

473 O’Hara AM, Shanahan F. (2006). The gut flora as a forgotten organ. EMBO Reports 7: 688-693.

474 Phillips CD, Phelan G, Dowd SE, McDonough MM, Ferguson AW, Delton Hanson J, et al. 475 (2012). Microbiome analysis among bats describes influences of host phylogeny, life history, 476 physiology and geography. Molecular Ecology 21: 2617-2627.

477 Phillips CD, Hanson J, Wilkinson JE, Koenig L, Rees E, Webala P, et al. (2017). Microbiome 478 Structural and Functional Interactions across Host Dietary Niche Space. Integrative and 479 Comparative Biology. 57: 743-755.

480 Rózalska B, Radzicki G, Sadowska B, Markowski J, Rudnicka W. (1998). Aerobic Microflora of 481 Myotis myotis (Borkhausen, 1797) and Barbastella barbastella (Schreber, 1774). Bulletin of the 482 Polish Academy of Sciences, Biological Sciences 46: 59-67.

483 Saraswati S, Sitaraman R. (2015). Aging and the human gut microbiota-from correlation to 484 causality. Frontiers in Microbiology 5: 764.

485 Shen Y-Y, Liang L, Zhu Z-H, Zhou W-P, Irwin DM, Zhang Y-P. (2010). Adaptive evolution of 486 energy metabolism genes and the origin of flight in bats. Proceedings of the National Academy of 487 Sciences 107 8666-8671.

488 Swanson KS, Dowd SE, Suchodolski JS, Middelbos IS, Vester BM, Barry KA, et al. (2011). 489 Phylogenetic and gene-centric metagenomics of the canine intestinal microbiome reveals 490 similarities with humans and mice. ISME Journal 5: 639-649.

491 Tung J, Barreiro LB, Burns MB, Grenier J-C, Lynch J, Grieneisen LE, et al (2015). Social 492 networks predict gut microbiome composition in wild baboons. eLife 4: e05224.

493 Van Tongeren SP, Slaets JPJ, Harmsen HJM, Welling GW. (2005). Fecal microbiota composition 494 and frailty. Applied Environmental Microbiology 71: 6438-6442.

495 Voigt CC, Speakman JR. (2007). Nectar-feeding bats fuel their high metabolism directly with 496 exogenous carbohydrates. Functional Ecology 21: 913-921.

497 Voigt CC, Baier L, Speakman JR, Siemers BM. (2008). Stable carbon isotopes in exhaled breath 498 as tracers for dietary information in birds and mammals. Journal of Experimental Biology 211: $4992233-2238$.

500 Voigt CC, Sörgel K, Dechmann DK. (2010). Refueling while flying: Foraging bats combust food 501 rapidly and directly to power flight. Ecology 91: 2908 - 2917.

502 Ward JH Jr. (1963). Hierarchical Grouping to Optimize an Objective Function. Journal of the 503 American Statistical Association 58: 236-244.

504 Weiss S, Xu ZZ, Peddada S, Amir A, Bittinger K, Gonzalez A, et al. (2017). Normalization and 505 microbial differential abundance strategies depend upon data characteristics. Microbiome 5: 27. 
506 Yatsunenko T, Rey FE, Manary MJ, Trehan I, Dominguez-Bello MG, Contreras M, et al. (2012). 507 Human gut microbiome viewed across age and geography. Nature 486: 222-227. 
Figure 1 (on next page)

Rarefaction curves of bat microbiomes.

Read data was rarefied to 8000 OTUs, and measured using phylogenetic diversity. (a) The alpha diversity of bat samples were clustered based on gender, to investigate differences in bacterial abundance between males and female. (b) Reads were clustered based on age datasets, with age dataset 2 displayed ( 0 yrs considered juvenile, $\geq 1$ yr considered adult). 


\section{PeerJ}

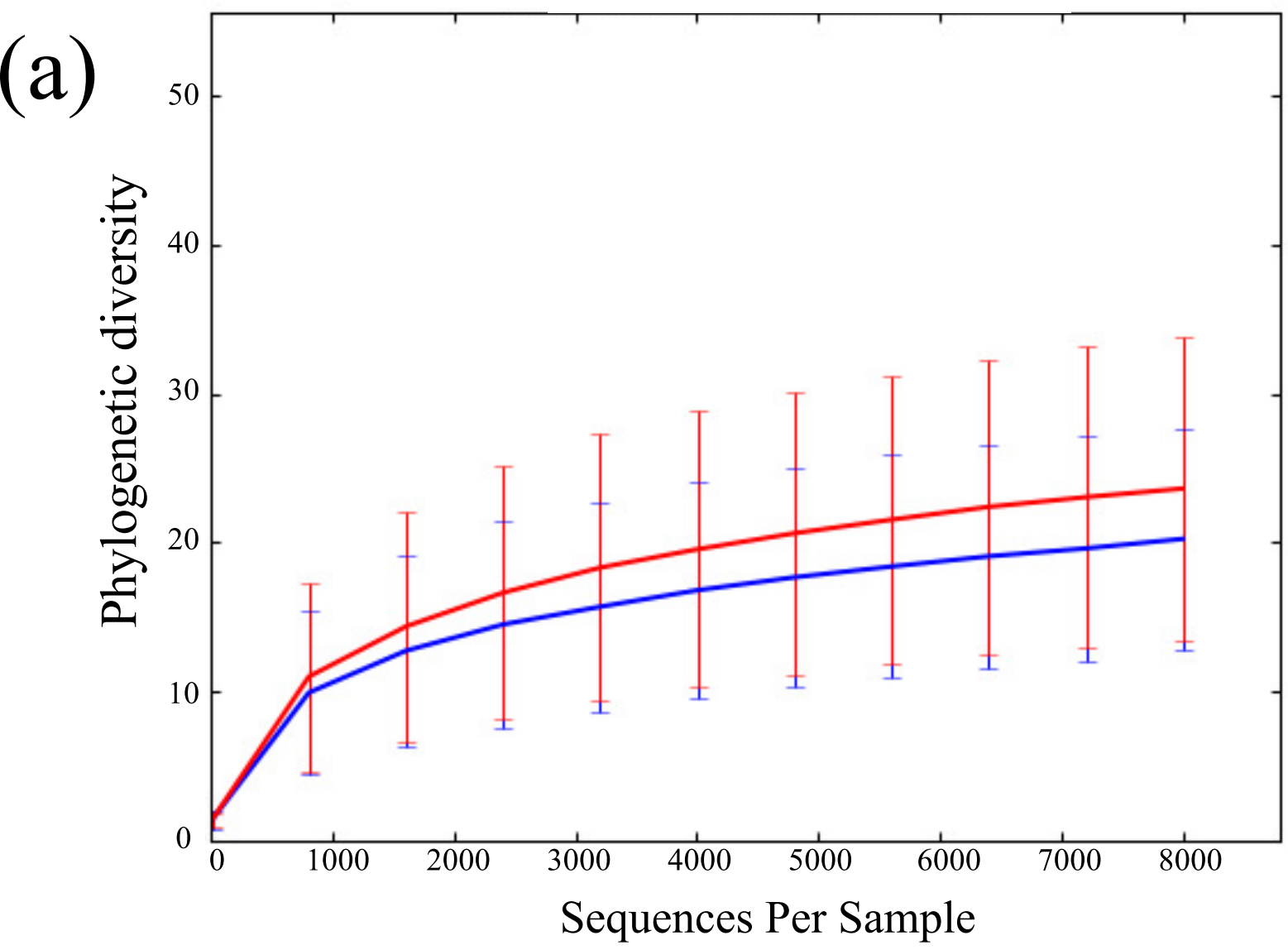

Female

Male

(b)

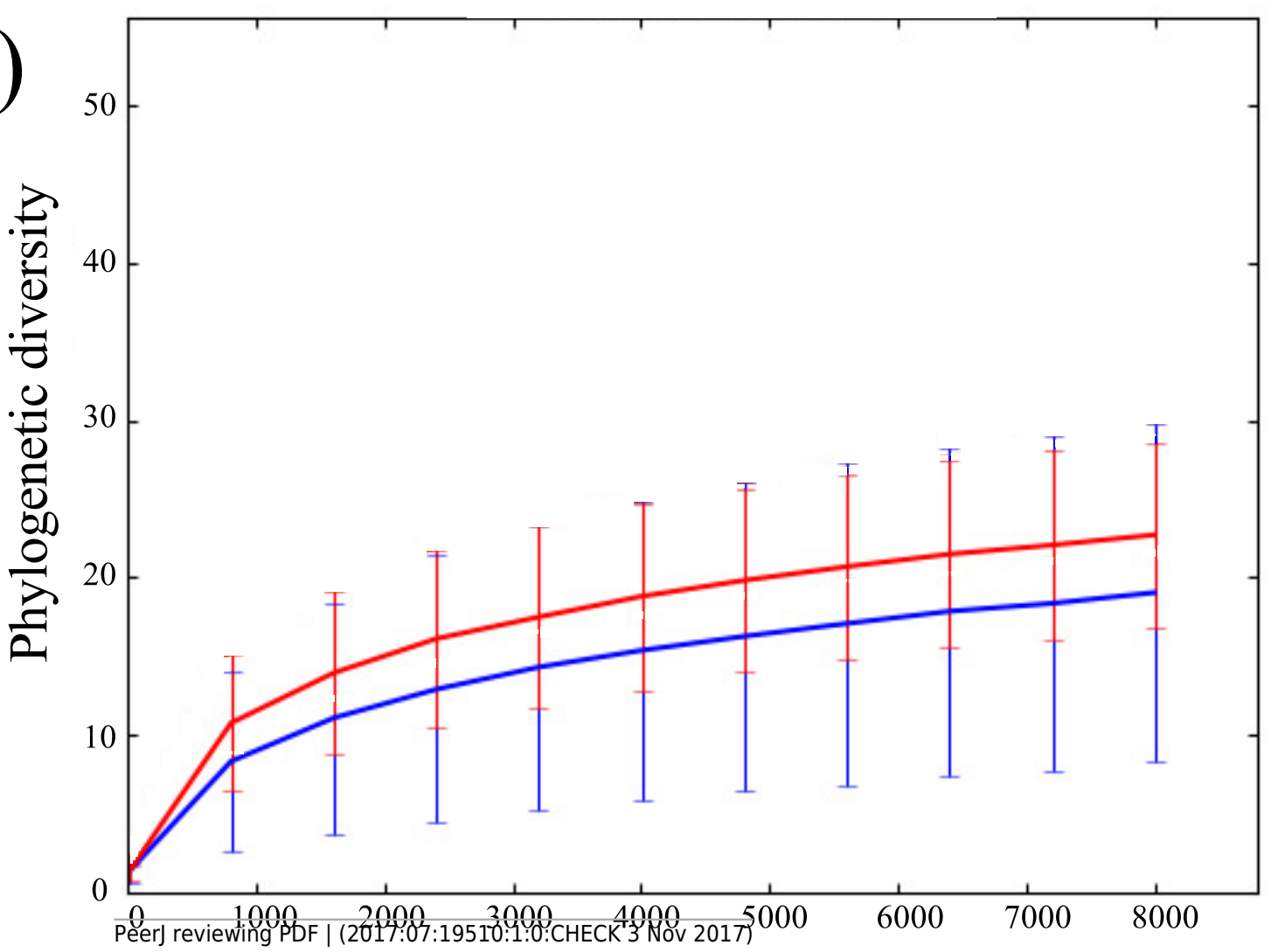


Figure 2 (on next page)

Principal components analysis of beta diversity.

Similarity based on diversity between samples was explored using PCoA after rarefaction, explaining $60.59 \%$ variance. (a) Beta diversity of male and female samples using weighted Unifrac distances are displayed. (b) Beta diversity using weighted Unifrac distances between adults and juveniles in age dataset 2 are displayed. 
(a) Gender

Peers

PC1 $(28.87 \%)$
Manuscript to be reviewed

Female

Male

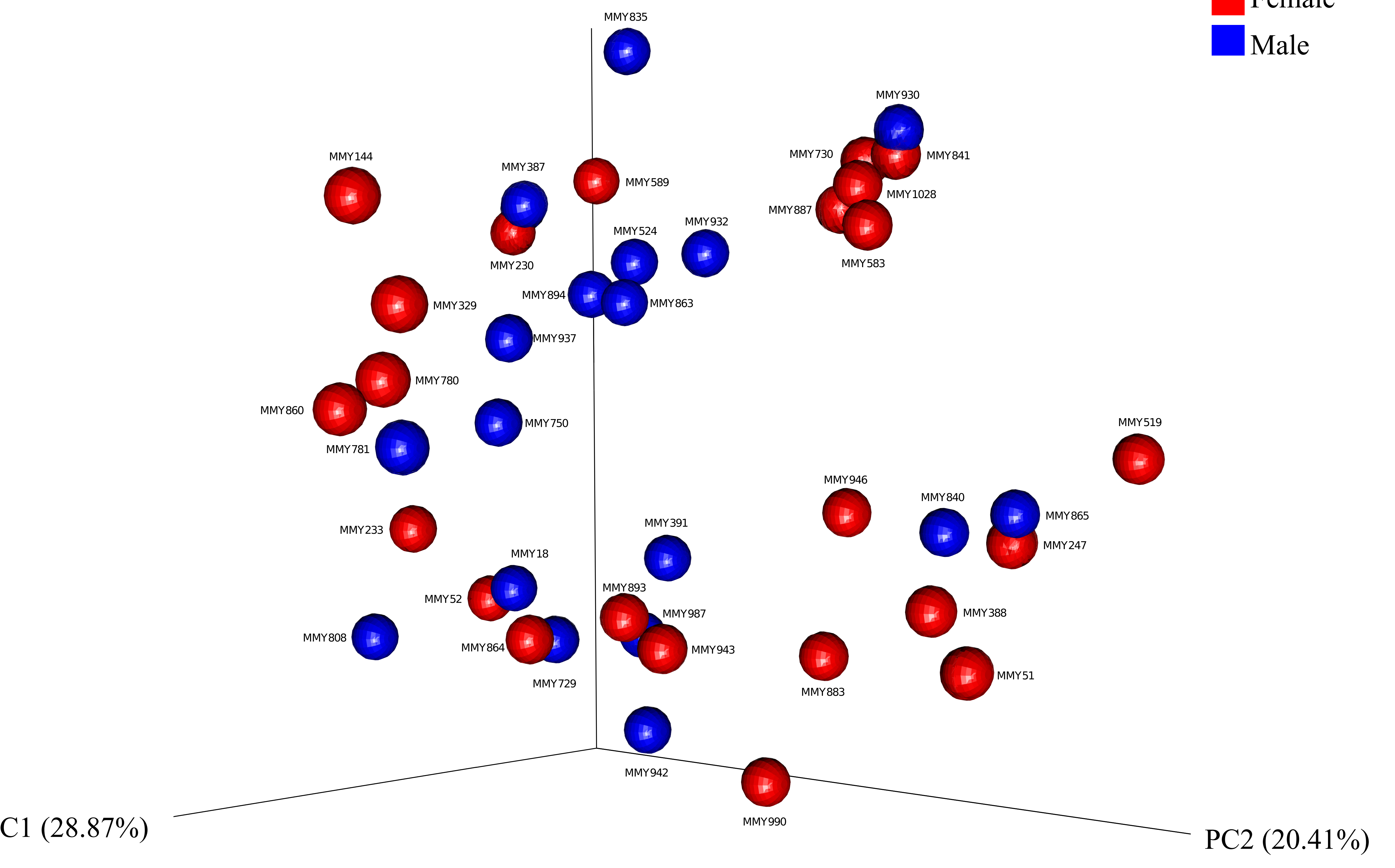

(b)

Age Bin 2

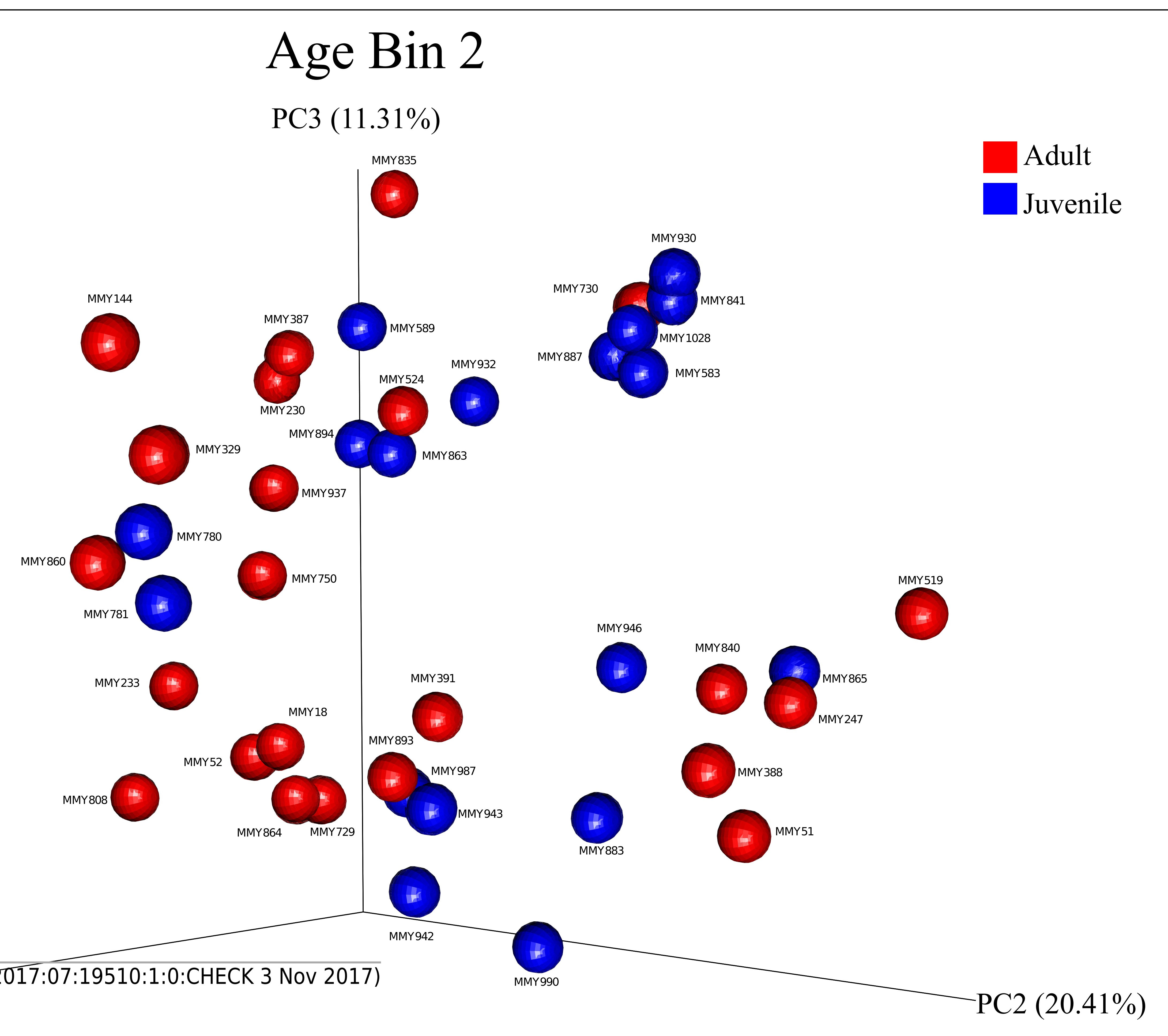


Figure 3 (on next page)

KEGG pathways present in the bat anal microbiota.

PICRUSt analyses of the bat anal microbiota identified a number of different KEGG gene pathways present. 


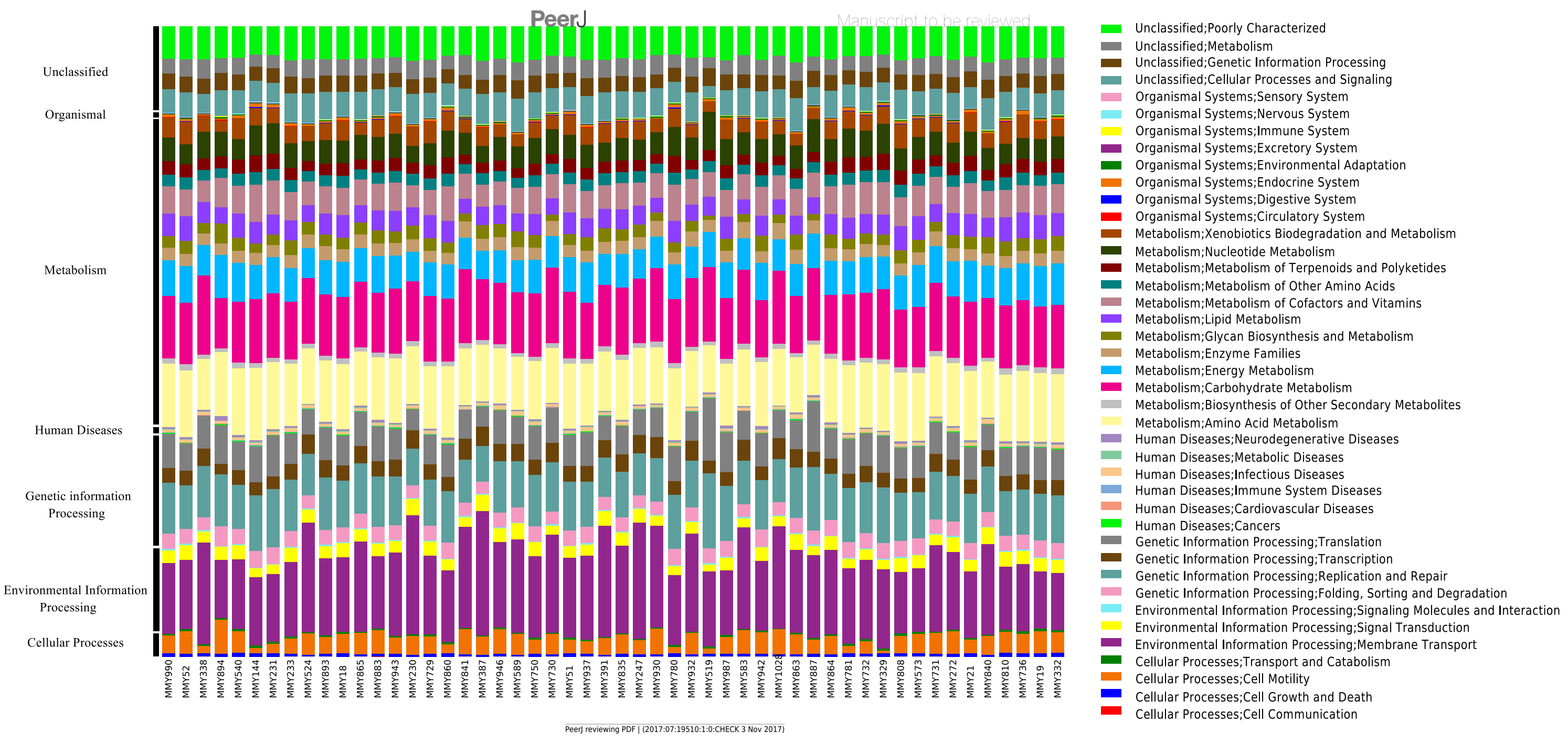




\section{Figure 4 (on next page)}

Phylogenetic comparison of microbiome bacterial abundance.

The most abundant bacterial phyla expressed in a range of diverse mammalian gut microbiome samples are compared and displayed using an established mammalian phylogeny. Bat samples from Carillo-Araujo et al. (2015) are identified with '2015'. Dietary niches are also displayed. 

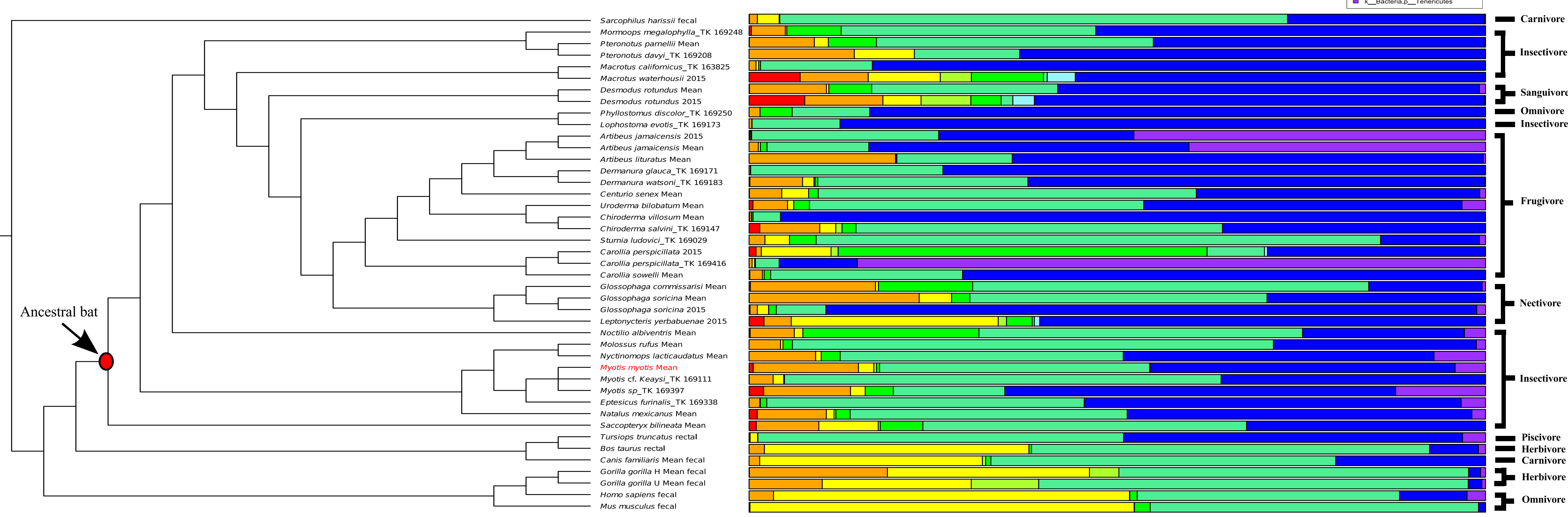


\section{Table $\mathbf{1}$ (on next page)}

Sample Information

Sample (MMY + sample number) metadata describing different numerical and categorical variables are displayed. The number of reads sequenced for each sample, and subsequent alpha diversity measures (PD Whole tree and observed OTUs) are counted. Age datasets were used to compare individual ages and to compare juveniles and adults. 


\begin{tabular}{|c|c|c|c|c|c|c|c|c|c|}
\hline \#Sample ID & Sex & Age Dataset 1 & Age Dataset 2 & Age Dataset 3 & Age Dataset 4 & Site & \# Reads & PD Whole Tree & Observed OTUs \\
\hline MMY1028 & $\mathrm{F}$ & 0 & Juvenile & Juvenile & 0 & LRB & 75,913 & 7.81 & 77 \\
\hline MMY144 & $\mathrm{F}$ & NA & Adult & Adult & $4+$ & Beg & 30,005 & 25.03 & 242 \\
\hline MMY18 & $\mathrm{M}$ & NA & Adult & NA & $1+$ & LRB & 136,568 & 28.16 & 421 \\
\hline MMY19 & $\mathrm{M}$ & NA & Adult & NA & $1+$ & LRB & 243 & 26.59 & 352 \\
\hline MMY21 & $\mathrm{F}$ & NA & Adult & Adult & $2+$ & LRB & 684 & 29.75 & 446 \\
\hline MMY230 & $\mathrm{F}$ & 3 & Adult & Adult & 3 & Fer & 43,623 & 15.28 & 184 \\
\hline MMY231 & $\mathrm{M}$ & NA & Adult & Adult & $3+$ & Fer & 1911 & 17.65 & 183 \\
\hline MMY233 & $\mathrm{F}$ & NA & Adult & Adult & $3+$ & Fer & 52,169 & 18.68 & 289 \\
\hline MMY247 & $\mathrm{F}$ & NA & Adult & Adult & $4+$ & Site undefined & 58,855 & 25.07 & 322 \\
\hline MMY272 & $\mathrm{F}$ & 1 & Adult & Juvenile & 1 & Fer & 275 & 31.98 & 470 \\
\hline MMY329 & $\mathrm{F}$ & 1 & Adult & Juvenile & 1 & Fer & 140,485 & 33.53 & 411 \\
\hline MMY332 & $\mathrm{M}$ & 0 & Juvenile & Juvenile & 0 & Fer & 97 & 17.14 & 182 \\
\hline MMY387 & $\mathrm{M}$ & NA & Adult & Adult & $2+$ & NM & 107,414 & 36.85 & 560 \\
\hline
\end{tabular}




\begin{tabular}{|c|c|c|c|c|c|c|c|c|c|}
\hline MMY388 & $\mathrm{F}$ & NA & Adult & NA & $1+$ & NM & 104,612 & 19.21 & 280 \\
\hline MMY391 & $\mathrm{M}$ & NA & Adult & Adult & $2+$ & NM & 42,732 & 10.92 & 114 \\
\hline MMY51 & $\mathrm{F}$ & NA & Adult & NA & $1+$ & Site undefined & 66,819 & 7.61 & 103 \\
\hline MMY519 & $\mathrm{F}$ & NA & Adult & NA & $1+$ & NM & 49,322 & 30.86 & 489 \\
\hline MMY52 & $\mathrm{F}$ & NA & Adult & NA & $1+$ & LRB & 10,971 & 15.55 & 141 \\
\hline MMY524 & $\mathrm{M}$ & 1 & Adult & Juvenile & 1 & NM & 52,755 & 22.97 & 313 \\
\hline MMY540 & $\mathrm{F}$ & NA & Adult & NA & $1+$ & NM & 586 & 32.86 & 441 \\
\hline MMY573 & $\mathrm{F}$ & NA & Adult & Adult & $4+$ & Beg & 134 & 27.65 & 350 \\
\hline MMY583 & $\mathrm{F}$ & 0 & Juvenile & Juvenile & 0 & Beg & 76,738 & 23.03 & 368 \\
\hline MMY589 & $\mathrm{F}$ & 0 & Juvenile & Juvenile & 0 & Beg & 128,039 & 21.09 & 179 \\
\hline MMY729 & $\mathrm{M}$ & 1 & Adult & Juvenile & 1 & Fer & 85,598 & 15.35 & 213 \\
\hline MMY730 & $\mathrm{F}$ & 1 & Adult & Juvenile & 1 & Fer & 165,854 & 14.01 & 108 \\
\hline MMY731 & $\mathrm{F}$ & NA & Adult & Adult & $2+$ & Fer & 305 & 23.40 & 315 \\
\hline MMY732 & $\mathrm{F}$ & NA & Adult & Adult & $3+$ & Fer & 1173 & 10.60 & 136 \\
\hline
\end{tabular}




\begin{tabular}{|c|c|c|c|c|c|c|c|c|c|}
\hline MMY736 & $\mathrm{M}$ & 3 & Adult & Adult & 3 & Fer & 52 & 30.48 & 428 \\
\hline MMY750 & $\mathrm{M}$ & NA & Adult & NA & $1+$ & Fer & 46,504 & 7.55 & 163 \\
\hline MMY780 & $\mathrm{F}$ & 0 & Juvenile & Juvenile & 0 & $\mathrm{NM}$ & 34,536 & 27.65 & 399 \\
\hline MMY781 & $\mathrm{M}$ & 0 & Juvenile & Juvenile & 0 & $\mathrm{NM}$ & 44,487 & 18.57 & 165 \\
\hline MMY808 & $\mathrm{M}$ & NA & Adult & Adult & $2+$ & $\mathrm{NM}$ & 79,976 & 38.83 & 554 \\
\hline MMY810 & $\mathrm{M}$ & NA & Adult & Adult & $2+$ & $\mathrm{NM}$ & 498 & 17.23 & 202 \\
\hline MMY835 & $\mathrm{M}$ & 1 & Adult & Juvenile & 1 & LRB & 91,311 & 9.32 & 84 \\
\hline MMY840 & $\mathrm{M}$ & NA & Adult & Adult & $2+$ & LRB & 55,681 & 10.791 & 137 \\
\hline MMY841 & $\mathrm{F}$ & 0 & Juvenile & Juvenile & 0 & LRB & 44,925 & 20.93 & 267 \\
\hline MMY860 & $\mathrm{F}$ & 1 & Adult & Juvenile & 1 & $\mathrm{NM}$ & 39,929 & 26.58 & 361 \\
\hline MMY863 & $\mathrm{M}$ & 0 & Juvenile & Juvenile & 0 & $\mathrm{NM}$ & 106,015 & 21.11 & 312 \\
\hline MMY864 & $\mathrm{F}$ & NA & Adult & Adult & $2+$ & $\mathrm{NM}$ & 9608 & 25.91 & 302 \\
\hline MMY865 & $\mathrm{M}$ & 0 & Juvenile & Juvenile & 0 & $\mathrm{NM}$ & 62,705 & 25.22 & 353 \\
\hline MMY883 & $\mathrm{F}$ & 0 & Juvenile & Juvenile & 0 & NM & 59,037 & 47.05 & 747 \\
\hline
\end{tabular}




\begin{tabular}{|c|c|c|c|c|c|c|c|c|c|}
\hline MMY887 & $\mathrm{F}$ & 0 & Juvenile & Juvenile & 0 & LRB & 99,393 & 7.81 & 77 \\
\hline MMY893 & $\mathrm{F}$ & NA & Adult & NA & $1+$ & NM & 56,760 & 25.03 & 242 \\
\hline MMY894 & $\mathrm{M}$ & 0 & Juvenile & Juvenile & 0 & NM & 82,872 & 28.16 & 421 \\
\hline MMY930 & $\mathrm{M}$ & 0 & Juvenile & Juvenile & 0 & LRB & 83,416 & 26.59 & 352 \\
\hline MMY932 & $\mathrm{M}$ & 0 & Juvenile & Juvenile & 0 & LRB & 191,120 & 29.75 & 446 \\
\hline MMY937 & $\mathrm{M}$ & 1 & Adult & Juvenile & 1 & NM & 12,983 & 15.28 & 184 \\
\hline MMY942 & $\mathrm{M}$ & 0 & Juvenile & Juvenile & 0 & NM & 60,449 & 17.65 & 183 \\
\hline MMY943 & $\mathrm{F}$ & 0 & Juvenile & Juvenile & 0 & NM & 15,264 & 18.68 & 289 \\
\hline MMY946 & $\mathrm{F}$ & 0 & Juvenile & Juvenile & 0 & NM & 118,085 & 25.07 & 322 \\
\hline MMY987 & M & 0 & Juvenile & Juvenile & 0 & NM & 25,295 & 31.98 & 470 \\
\hline MMY990 & $\mathrm{F}$ & 0 & Juvenile & Juvenile & 0 & NM & 93,196 & 33.53 & 411 \\
\hline
\end{tabular}




\section{Table 2 (on next page)}

Phylogenetic diversity within the Myotis myotis microbiome

Mean Phylogenetic Diversity (PD) for each category, representing the range of bacterial species present, are shown for each data category. 


\begin{tabular}{|c|c|c|c|c|c|c|c|}
\hline \multirow{2}{*}{$\begin{array}{c}\text { Age } \\
\text { dataset } 1\end{array}$} & 0 Years & 1 Years & 3 Years & Unknown & & & \\
\hline & 19.36 & 21.24 & 26.58 & 26.14 & & & \\
\hline \multirow{2}{*}{$\begin{array}{c}\text { Age } \\
\text { dataset } 2 \\
\end{array}$} & Adults & Juveniles & & & & & \\
\hline & 24.67 & 19.36 & & & & & \\
\hline & & & & & & & \\
\hline \multirow{2}{*}{$\begin{array}{c}\text { Age } \\
\text { dataset } 3 \\
\end{array}$} & Adults & Juveniles & Unknown & & & & \\
\hline & 24.01 & 19.88 & 28.94 & & & & \\
\hline \multirow{3}{*}{$\begin{array}{c}\text { Age } \\
\text { dataset } 4\end{array}$} & & & & & & & \\
\hline & 0 Years & 1 Year & $1+$ Years & $2+$ Years & 3 Years & 3+ Years & 4+ Years \\
\hline & 19.36 & 21.24 & 28.94 & 23.90 & 26.59 & 29.75 & 20.15 \\
\hline \multirow{2}{*}{ Gender } & Male & Female & & & & & \\
\hline & 20.4 & 23.85 & & & & & \\
\hline & & & & & & & \\
\hline \multirow{2}{*}{ Site } & Beg & Fer & LRB & NM & & & \\
\hline & 14.52 & 23.89 & 18.00 & 24.67 & & & \\
\hline
\end{tabular}




\section{Table 3 (on next page)}

Core microbiomes

Bacteria phyla and the number of OTUs present in a range of core microbiome percentage thresholds for M. myotis are displayed. 


\begin{tabular}{|l|c|c|c|c|c|}
\hline & Core 50\% & Core $60 \%$ & Core $70 \%$ & Core $80 \%$ & Core $90 \%$ \\
\hline Number of OTUs & 47 & 29 & 20 & 13 & 7 \\
\hline$\%$ Proteobacteria & $57.45 \%$ & $55.17 \%$ & $50 \%$ & $38.46 \%$ & $42.86 \%$ \\
\hline$\%$ Actinobacteria & $17.02 \%$ & $17.24 \%$ & $20 \%$ & $15.38 \%$ & $14.28 \%$ \\
\hline$\%$ Firmicutes & $17.02 \%$ & $20.69 \%$ & $30 \%$ & $46.16 \%$ & $42.86 \%$ \\
\hline$\%$ Cyanobacteria & $4.25 \%$ & $3.45 \%$ & $0 \%$ & $0 \%$ & $0 \%$ \\
\hline$\%$ Chlamydiae & $2.13 \%$ & $0 \%$ & $0 \%$ & $0 \%$ & $0 \%$ \\
\hline$\%$ Tenericutes & $2.13 \%$ & $3.45 \%$ & $0 \%$ & $0 \%$ & $0 \%$ \\
\hline
\end{tabular}

$$
1
$$

